\title{
DC Glow Discharge in Axial Magnetic Field at Low Pressures
}

\author{
Shen Gao, ${ }^{1}$ Shixiu Chen, ${ }^{1}$ Zengchao Ji, ${ }^{1}$ Wei Tian, ${ }^{2}$ and Jun Chen ${ }^{1,3}$ \\ ${ }^{1}$ School of Electrical Engineering, Wuhan University, Bayi Road 299, Hubei, Wuhan 430072, China \\ ${ }^{2}$ College of Computer Science, South-Central University for Nationalities, National Avenue 182, Hubei, Wuhan 430074, China \\ ${ }^{3}$ School of Electrical and Electronic Engineering, Hubei University of Technology, Nan Li Road 28, Hubei, Wuhan 430068, China
}

Correspondence should be addressed to Shixiu Chen; sxiuchen@163.com

Received 29 January 2017; Revised 10 April 2017; Accepted 4 May 2017; Published 28 May 2017

Academic Editor: Alkesh Punjabi

Copyright (C) 2017 Shen Gao et al. This is an open access article distributed under the Creative Commons Attribution License, which permits unrestricted use, distribution, and reproduction in any medium, provided the original work is properly cited.

\begin{abstract}
On the basis of fluid approximation, an improved version of the model for the description of dc glow discharge plasma in the axial magnetic field was successfully developed. The model has yielded a set of analytic formulas for the physical quantities concerned from the electron and ion fluids equations and Poisson equation. The calculated results satisfy the practical boundary conditions. Results obtained from the model reveal that although the differential equations under the condition of axial magnetic field are consistent with the differential equations without considering the magnetic field, the solution of the equations is not completely consistent. The results show that the stronger the magnetic field, the greater the plasma density.
\end{abstract}

\section{Introduction}

Low pressure DC glow discharge plasma technology is applied widely in many fields, like plasma etching, plasma material surface treatment, plasma electron beam source, plasma sputtering spraying, and so on [1-3]. Many scholars have carried out various studies on low pressure glow discharge experimentally and theoretically. In the experimental study, a variety of means have been employed, such as electrostatic probe detection and spectroscopic diagnostic methods to analyze and study physical processes and discharge mechanism of low pressure glow discharge [3-6].

Despite the fact that the influence of the magnetic field on the parameters of the plasma has been studied in the experiment, in theoretical research, there is still not a complete theoretical description of the influence of magnetic field on the parameters of the glow discharge. The relations between the physical quantities and some physical phenomena can not be explained strictly. Most authors use numerical methods in the theoretical study [7-14]. With the development of computer and algorithm optimization, these methods provide a good foundation and guarantee with great achievements made in this field. However, seen from previous numerical settlement results, there are also common problems: (1) numerical method applies some imaginary boundary conditions in the calculation process, which often leads to unreasonable results; for example, it can not be self-consistent; (2) unstabilizing factor may occur during the calculation, which leads to phenomenon of divergence, so calculation has to stop halfway; (3) in the lengthy process of calculation, it is not easy to find human error and check calculation results. Moreover, relationship between the various physical quantities is not readily apparent. In order to avoid these shortcomings, some of the early researchers sought analytic expression of the relevant parameters from the relevant classic discharge equation with applied analytic methods and actual boundary conditions and the theoretical model of glow discharge plasma under no magnetic field is established [15-19].

The present study, as a continuation of the previous work, is an attempt to improve the theoretical formulation mainly by considering the influence of axial magnetic field on the glow discharge plasma. The specific objective of this study is to obtain a set of analytic expressions that can describe the glow discharge plasma in the axial magnetic field on the whole discharge area. The final goal is to gain the relationship between the plasma parameters and the axial magnetic field. In the following presentation, we shall first 


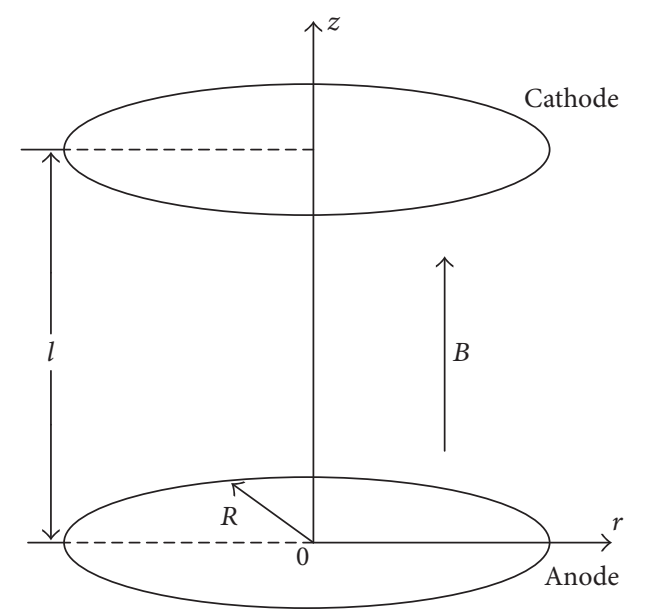

FIGURE 1: Schematic of a glow discharge with external magnetic field.

discuss the aspects concerning the basic assumptions and theoretical formulation of the physical model for the glow discharge plasma in axial magnetic field. The computational results and detailed mathematical analysis shall be presented in the subsequent sections. In the final part, the above results are analyzed and corresponding conclusions are drawn.

\section{Physical Model}

In this study, the two-dimensional dc discharge configuration is shown in Figure 1. The powered electrode is the anode and the grounded one is the cathode. In this theoretical formulation, cylindrical coordinates $r$ and $z$ are used for the basic discharge equations, the coordinate $\theta$ in this case is irrelevant as the plasma is taken to be axially symmetrical.

The model is based on the following assumptions:

(1) The direction of the applied magnetic field is parallel to the direction of the electric field. The electric field is produced by the voltage at both ends of the anode and cathode. Edge effects of electric field are neglected.

(2) Plasma formation is mainly a result of electrons collision with neutral atoms, while generation of negative ions and other factors are not considered.

(3) It is generally considered that in low-temperature glow discharge plasma model, ions maintain the same temperature as neutral gas. Hence, there is no need to consider energy equation $[15,16]$.

(4) Generally, glow discharge current is about several milliamps. Self-magnetic field generated by glow current is much smaller than applied external magnetic field. Therefore, effect of self-magnetic field was ignored and only effect of external magnetic field on glow discharge was considered.
The basic equations employed in this theoretical formulation are the particle and momentum conservation equations coupled with one of the Maxwell equations.

$$
\begin{aligned}
& \frac{\partial n_{\alpha}}{\partial t}+\nabla \cdot\left(n_{\alpha} u_{\alpha}\right)=\xi_{i} n_{e} . \\
& m_{\alpha} n_{\alpha}\left\{\frac{\partial}{\partial t}+u_{\alpha} \cdot \nabla\right\} u_{\alpha} \\
& =q_{\alpha} n_{\alpha}\left(E+u_{\alpha} \times B\right)-\nabla\left(k T_{\alpha} n_{\alpha}\right)-m_{\alpha} u_{\alpha} n_{\alpha} \nu_{\alpha n} \\
& \nabla \cdot E=\frac{e}{\varepsilon_{0}} \sum_{\alpha=i, e} n_{\alpha} .
\end{aligned}
$$

In the above equations the various notations are defined as follows: $\xi_{i}$ is the ionization frequency; subscript $\alpha$ represent electron or ion; $n_{\alpha}, u_{\alpha}, m_{\alpha}$, and $T_{\alpha}$ are, respectively, the number density, average velocity, mass, and temperature; $v_{\alpha n}$ is the respective collision frequency of electrons and positive ions with neutral particles and they are assumed to be constants in this case; $E$ is the electric field; $B$ is the magnetic field; $q_{\alpha}$ is the electronic charge and $\varepsilon_{0}$ is the permittivity constant; $k$ is the Boltzmann constant.

We shall first consider these equations for a steady state case in which all the terms involving time derivative vanish. Next, we neglect the inertial terms $\left\{u_{\alpha} \cdot \nabla\right\} u_{\alpha}$ in (2), as in the present case the average flow velocity in general is much smaller than the average speed of the random thermal motions [18]. Equation (2) becomes the following form:

$$
q_{\alpha} E+q_{\alpha}\left(u_{\alpha} \times B\right)-\frac{T_{\alpha}}{n_{\alpha}} \nabla n_{\alpha}=m_{\alpha} \nu_{\alpha n} u_{\alpha} .
$$

According to the model assumptions (1), electric field is axial component, $E_{\theta}=E_{r}=0$ and $B_{\theta}=B_{r}=0$. Taking into 
account (3), conditions assumed by the model, component form of (4) parallel to magnetic field is

$$
u_{\alpha \|}=b_{\alpha \|} E_{z}-D_{\alpha \|} \frac{\nabla_{\|} n_{\alpha}}{n_{\alpha}},
$$

where

$$
\begin{gathered}
b_{\alpha \|}=\frac{q_{\alpha}}{m_{\alpha} v_{\alpha n}} \\
D_{\alpha \|}=\frac{k T_{\alpha}}{m_{\alpha} \nu_{\alpha n}} .
\end{gathered}
$$

In the direction perpendicular to the magnetic field, (4) is

$$
u_{\alpha \perp}=-D_{\alpha \perp} \frac{\nabla_{\perp} n_{\alpha}}{n_{\alpha}}-D_{\alpha d} \frac{\nabla_{\perp} n_{\alpha}}{n_{\alpha}} \times b_{0}
$$

where

$$
\begin{aligned}
D_{\alpha \perp} & =\frac{v_{\alpha n} k T_{\alpha}}{m_{\alpha}\left(\omega_{\alpha}^{2}+v_{\alpha n}^{2}\right)} \\
D_{\alpha d} & =\frac{k T_{\alpha}}{q_{\alpha} B\left(1+v_{\alpha n}^{2} / \omega_{\alpha}^{2}\right)} \\
\omega_{\alpha} & =\frac{q_{\alpha} B}{m_{\alpha}} .
\end{aligned}
$$

The plasma is assumed to be uniformly distributed in the angular direction and we obtained

$$
\begin{aligned}
\nabla \cdot\left(n_{\alpha} u_{\alpha}\right)= & b_{\alpha \|} n_{\alpha} \frac{\partial E}{\partial z}+b_{\alpha \|} E \frac{\partial n_{\alpha}}{\partial z}-D_{\alpha \|} \frac{\partial^{2} n_{\alpha}}{\partial z^{2}} \\
& -D_{\alpha \perp} \frac{\partial^{2} n_{\alpha}}{\partial r^{2}}-D_{\alpha \perp} \frac{1}{r} \frac{\partial n_{\alpha}}{\partial r} .
\end{aligned}
$$

Ionization frequency can be expressed as follows:

$$
\zeta_{i}=\beta_{1} u_{E}+\beta_{2} u_{D}
$$

where $u_{E}$ is the electron drift velocity along the axial direction and $u_{D}$ is the electron diffusion velocity. $\beta_{2}$ is a pressure dependent constant to be determined. $\beta_{1}$ is the Townsend ionization coefficient and is a function of the gas pressure $p$ and electric field $E$. In the case of low pressure and high electric field strength, this implies that $B_{c} p / E \ll 1$ and $\beta_{1}$ can be Taylor expansion.

$$
\beta_{1}=A p \exp \left(-\frac{B_{c} p}{E}\right) \approx A p-\frac{A B_{c} p^{2}}{E}
$$

$A$ and $B_{c}$ are constants related to gases.
From (1), (3), (5), (7), (11), (12), and (13), we obtain

$$
\begin{aligned}
& \left(-b_{e \|} n_{e} \frac{\partial E}{\partial z}-b_{e \|} E \frac{\partial n_{e}}{\partial z}+A p b_{e \|} E n_{e}\right) \\
& -\left(D_{e \perp} \frac{\partial^{2} n_{e}}{\partial r^{2}}+D_{e \perp} \frac{1}{r} \frac{\partial n_{e}}{\partial r}\right) \\
& +\left(-D_{e \|} \frac{\partial^{2} n_{e}}{\partial z^{2}}+D_{e \|} \beta_{2} \frac{\partial n_{e}}{\partial z}-A B_{c} p^{2} b_{e \|} n_{e}\right)=0 \\
& \left(b_{i \|} n_{i} \frac{\partial E}{\partial z}+b_{i \|} E \frac{\partial n_{i}}{\partial z}+A p b_{e \|} E n\right) \\
& -\left(D_{i \perp} \frac{\partial^{2} n_{i}}{\partial r^{2}}+D_{i \perp} \frac{1}{r} \frac{\partial n_{i}}{\partial r}\right)_{e} \\
& +\left(-D_{i \|} \frac{\partial^{2} n_{i}}{\partial z^{2}}-A B_{c} p^{2} b_{e \|} n_{e}+D_{e \|} \beta_{2} \frac{\partial n_{e}}{\partial z}\right)=0 \\
& \frac{\partial E}{\partial z}=\frac{e}{\varepsilon_{0}}\left(n_{i}-n_{e}\right) .
\end{aligned}
$$

Equations (14), (15), and (16) constitute final form of glow discharge plasma physics model equation under axial magnetic field condition. In the above equations, except $n_{e}$, $n_{i}$, and $E$, all other parameters can be regarded as constants in calculation. Equations (14), (15), and (16) are consistent with the model of the glow discharge without considering the axial magnetic field [18]. Their difference is only the difference in parameters. But it is not possible to apply the expression of the glow discharge model without considering the axial magnetic field into (14), (15), and (16) as this can appear as imaginary solutions. In the absence of magnetic field, the transport coefficient of the lighter particles is larger than that of the heavy particles. This conclusion is contrary to the case of magnetic field. The axial magnetic field has a restraining effect on the transverse motion of charged particles. The radius of gyration of the ion is much larger than that of the electron. Therefore, the transverse transport coefficient of ions is larger than that of electrons. In the calculation process, due to the change of the transport coefficient, the form of the solution has changed accordingly. In the next section, we deduce the above equations again according to the similar derivation process of the earlier articles. The solutions obtained shall be exact and completely satisfy equations and the actual boundary conditions. The effect of magnetic field on the glow discharge plasma is analyzed.

\section{Mathematical Calculation}

We employ the variables separation method by writing

$$
n_{e}=Z(z) R(r)
$$

and letting

$$
A B_{c} p^{2} b_{e \|}=a+b+c,
$$


where $a, b$, and $c$ are constants. Substituting (17) and (18) into (14) and dividing it by (17), we obtain

$$
\begin{array}{r}
D_{e \|}\left(\frac{1}{Z} \frac{\partial^{2} Z}{\partial z^{2}}-\beta_{2} \frac{1}{Z} \frac{\partial Z}{\partial z}\right)+b=0 \\
D_{e \perp}\left(\frac{1}{R(r)} \frac{\partial^{2} R(r)}{\partial r^{2}}+\frac{1}{R(r)} \frac{1}{r} \frac{\partial R(r)}{\partial r}\right)+a=0 \\
b_{e \|}\left(\frac{\partial E}{\partial z}+\frac{E}{n_{e}} \frac{\partial n_{e}}{\partial z}-A p E\right)+c=0 .
\end{array}
$$

Equation (19) involves $z$ alone and (20) involves $r$ alone; (21) involves $r$ and $z$. The solution of (19) and (20) is

$$
\begin{aligned}
& Z(z)=\exp \left(\frac{\beta_{2}}{2} z\right)\left(C_{1} \exp \left(a_{1} z\right)+C_{2} \exp \left(-a_{1} z\right)\right) \\
& R(r)=J_{0}\left(\sqrt{\frac{a}{D_{e \perp}} r}\right),
\end{aligned}
$$

where

$$
a_{1}=\frac{1}{2} \sqrt{\beta_{2}^{2}-\frac{4 b}{D_{e \|}}}
$$

$$
\begin{aligned}
n^{\prime} & =\frac{\varepsilon_{0}}{e} \frac{\partial E}{\partial z}=-\frac{\varepsilon_{0}}{e} \frac{c}{b_{e \|}}\left\{\frac{A p \exp (A p z)}{\left(C_{1} \exp \left[\left(\beta_{2} / 2+a_{1}\right) z\right]+C_{2} \exp \left[\left(\beta_{2} / 2-a_{1}\right) z\right]\left[\left(\beta_{2} / 2-a_{1}\right) z\right]\right)}\right. \\
& \left.-\frac{\exp (A p z)\left(C_{1}\left(\beta_{2} / 2+a_{1}\right) \exp \left[\left(\beta_{2} / 2+a_{1}\right) z\right]+C_{2}\left(\beta_{2} / 2-a_{1}\right) \exp \left[\left(\beta_{2} / 2-a_{1}\right) z\right]\right)}{\left(C_{1} \exp \left[\left(\beta_{2} / 2+a_{1}\right) z\right]+C_{2} \exp \left[\left(\beta_{2} / 2-a_{1}\right) z\right]\right)^{2}}\right\} \\
& \cdot\left\{\frac{C_{1} \exp \left[\left(\beta_{2} / 2-A p+a_{1}\right) z\right]}{\left(\beta_{2} / 2-A p+a_{1}\right)}+\frac{C_{2} \exp \left[\left(\beta_{2} / 2-A p-a_{1}\right) z\right]}{\left(\beta_{2} / 2-A p-a_{1}\right)}+C_{3}\right\}-\frac{\varepsilon_{0}}{e} \frac{c}{b_{e}} .
\end{aligned}
$$

Substituting (27) into (15) and using (18), (19), (20), and (21), we obtain

$$
\begin{gathered}
\left(a\left(\frac{D_{i \perp}}{D_{e \perp}}-1\right)+b\left(\frac{D_{i \|}}{D_{e \|}}-1\right)-c\left(\frac{b_{i \|}}{b_{e \|}}+1\right)\right. \\
\left.+\left(D_{e \|}-D_{i \|}\right) \frac{\beta_{2}}{n_{e}} \frac{\partial n_{e}}{\partial z}+\operatorname{ApE}\left(b_{i \|}+b_{e \|}\right)\right) n_{e} \\
-\left\{D_{i \|}\left(\frac{\partial^{2} n^{\prime}}{\partial z^{2}}\right)-b_{i \|}\left(n^{\prime} \frac{\partial E}{\partial z}+E \frac{\partial n^{\prime}}{\partial z}\right)\right\}=0 .
\end{gathered}
$$

In (29), the first brace involves $z$ and $r$ and the second brace involves $z$ alone, so we have

Combining (22) and (23), we obtain

$$
\begin{aligned}
n_{e}(z, r)= & \exp \left(\frac{\beta_{2}}{2} z\right)\left(C_{1} \exp \left(a_{1} z\right)+C_{2} \exp \left(-a_{1} z\right)\right) \\
& \cdot J_{0}\left(\sqrt{\frac{a}{D_{e \perp}} r}\right) .
\end{aligned}
$$

Substituting (25) into (21), we have

$$
\begin{aligned}
E(z) & =-\frac{c}{b_{e \|}} \\
\cdot & \frac{\exp (A p z)}{\exp \left(\left(\beta_{2} / 2\right) z\right)\left(C_{1} \exp \left(a_{1} z\right)+C_{2} \exp \left(-a_{1} z\right)\right)} \\
& \cdot\left\{\frac{C_{1} \exp \left[\left(\beta_{2} / 2-A p+a_{1}\right) z\right]}{\left(\beta_{2} / 2-A p+a_{1}\right)}\right. \\
& \left.+\frac{C_{2} \exp \left[\left(\beta_{2} / 2-A p-a_{1}\right) z\right]}{\left(\beta_{2} / 2-A p-a_{1}\right)}+C_{3}\right\},
\end{aligned}
$$

where

$$
n_{i}=n^{\prime}+n_{e}
$$


Substituting (24), (25), (26), and (32) into (30), to make the variable $z$ within a certain range, can make (30) established; we have

$$
\begin{gathered}
a\left(\frac{D_{i \perp}}{D_{e \perp}}-1\right)+b\left(\frac{D_{i \|}}{D_{e \|}}-1\right)-c\left(\frac{b_{i \|}}{b_{e \|}}+1\right) \\
+\left(D_{e \|}-D_{i \|}\right) \frac{\beta_{2}}{n_{e}} \frac{\partial n_{e}}{\partial z}+A p E\left(b_{i \|}+b_{e \|}\right)=0 \\
{\left[\left(D_{e \|}-D_{i \|}\right) a_{1} \beta_{2}-\frac{c}{b_{e \|} a_{1}} A p\left(b_{i \|}+b_{e \|}\right)\right]} \\
\quad \cdot \frac{C_{1} \exp \left(a_{1} z\right)-C_{2} \exp \left(-a_{1} z\right)}{C_{1} \exp \left(a_{1} z\right)+C_{2} \exp \left(-a_{1} z\right)}=0 .
\end{gathered}
$$

Equation (33) involves $z$ and $r$ and (34) involves $z$ alone. Using (33) and (34), we have

$$
\begin{aligned}
a_{1} & =\sqrt{A^{2} p^{2}+a \frac{\left(D_{i \perp}-D_{e \perp}\right)}{D_{e \perp}\left(D_{e \|}-D_{i \|}\right)}} \\
\frac{D_{e \|}}{D_{i \|}} & =2+\frac{b_{e \|}}{b_{i \|}} .
\end{aligned}
$$

Using (32), (26) is simplified as

$$
E(z)=\frac{-2 D_{i \|} a_{1}}{b_{i \|}} \frac{C_{1} \exp \left(a_{1} z\right)-C_{2} \exp \left(-a_{1} z\right)}{C_{1} \exp \left(a_{1} z\right)+C_{2} \exp \left(-a_{1} z\right)} .
$$

The relationship between electric field and voltage is

$$
-\frac{d V}{d z}=E
$$

Using (38), we have

$$
V=\frac{2 D_{i \|}}{b_{i \|}} \ln \left[C_{1} \exp \left(a_{1} z\right)+C_{2} \exp \left(-a_{1} z\right)\right]+C_{4}
$$

In this paper, the physical model in magnetic field is consistent with the physical model without magnetic field. Therefore, the same boundary conditions can be applied to the physical model without magnetic field [18].

$$
\begin{gathered}
z=0, \\
r=0, \\
V=V_{0} .
\end{gathered}
$$

$$
\begin{aligned}
z & =l, \\
r & =0, \\
V & =0 \\
z & =0, \\
r & =0 \\
n_{e} & =n_{a} \\
z & =0 \\
r & =R, \\
n_{e} & =0 .
\end{aligned}
$$

Using boundary conditions (39), (40), (41), (42), and (25), we have

$$
\begin{aligned}
& C_{1}=n_{a} \frac{\exp \left(-a_{1} l\right)-\exp \left(-V_{0} b_{i \|} / 2 D_{i \|}\right)}{\exp \left(-a_{1} l\right)-\exp \left(a_{1} l\right)} \\
& C_{2}=n_{a} \frac{\exp \left(-V_{0} b_{i \|} / 2 D_{i \|}\right)-\exp \left(a_{1} l\right)}{\exp \left(-a_{1} l\right)-\exp \left(a_{1} l\right)} .
\end{aligned}
$$

Substituting (43) into (25), we have

$$
a=\left(\frac{2.405}{R}\right)^{2} D_{e \perp}
$$

That means the constants $\beta_{2}, C_{1}, C_{2}, a_{1}, a, b$, and $c$ have been completely determined. Through the solution of the above equation, we can analyze the influence of axial magnetic field on glow discharge. However, it should be noted that each parameter in the expressions must satisfy the following equation

$$
\begin{gathered}
A B_{c} p^{2} b_{e \|}=\left(\frac{2.405}{R}\right)^{2} D_{e \perp}-D_{e \|}\left(\frac{2.405}{R}\right)^{2} \\
\cdot \frac{\left(D_{i \perp}-D_{e \perp}\right)}{\left(D_{e \|}-D_{i \|}\right)}+2\left(D_{e \|}-D_{i \|}\right) \\
\cdot\left(A^{2} p^{2}+\left(\frac{2.405}{R}\right)^{2} \frac{\left(D_{i \perp}-D_{e \perp}\right)}{\left(D_{e \|}-D_{i \|}\right)}\right)
\end{gathered}
$$

Glow discharge current density is as follows

$$
\begin{aligned}
j & =j_{z}+j_{r}+j_{\theta} . \\
j_{z} & =e\left(n_{i} u_{i z}-n_{e} u_{e z}\right) \\
& =e\left[n_{i} b_{i \|} E_{z}-n_{e} b_{e \|} E_{z}+D_{e \|} \frac{\partial n_{e}}{\partial z}-D_{i \|} \frac{\partial n_{i}}{\partial z}\right] \\
j_{r} & =e\left(n_{i} u_{i r}-n_{e} u_{e r}\right)=e\left(D_{e \perp}-D_{i \perp}\right) \frac{\partial n_{e}}{\partial r} \\
j_{\theta} & =e\left(D_{i d}-D_{e d}\right) \frac{\partial n_{e}}{\partial r} .
\end{aligned}
$$


Equations (48), (49), and (50) satisfy the continuity equation of constant current

$$
\nabla \cdot j=0 .
$$

Using the above results, the glow discharge current can be obtained.

$$
\begin{aligned}
I_{z} & =\int_{0}^{R} J_{z} \cdot 2 \pi r \cdot d r=\left[\left(C_{1}\left(b_{i \|}-b_{e \|}\right)\left(\frac{-2 D_{i \|} a_{1}}{b_{i \|}}\right)\right.\right. \\
& \left.+C_{1}\left(D_{e \|}-D_{i \|}\right)\left(A p+a_{1}\right)\right) \exp \left[\left(A p+a_{1}\right) z\right] \\
& +\left(C_{2}\left(D_{e \|}-D_{i \|}\right)\left(A p-a_{1}\right)\right. \\
& \left.\left.-C_{2}\left(b_{i \|}-b_{e \|}\right)\left(\frac{-2 D_{i \|} a_{1}}{b_{i \|}}\right) \exp \left[\left(A p-a_{1}\right) z\right]\right)\right] \\
& \cdot\left(\frac{2 \pi e R^{2}}{2.405}\right) J_{1}(2.405) \\
I_{r} & =\int_{0}^{L} J_{r} \cdot 2 \pi r \cdot d z=2 \pi r e\left(\frac{2.405}{R}\right)\left(D_{i \perp}-D_{e \perp}\right) \\
& \cdot J_{1}\left(\frac{2.405}{R} r\right) \cdot \frac{C_{1}}{A p+a_{1}}\left\{\exp \left[\left(A p+a_{1}\right) L\right]-1\right\} \\
& +\frac{C_{2}}{A p-a_{1}}\left\{\exp \left[\left(A p-a_{1}\right) L\right]-1\right\} .
\end{aligned}
$$

In glow discharge experiments, the glow current is relatively easy to measure. Through (52), the relationship between the macrophysical parameters will be reflected.

\section{Result Analysis}

In the absence of magnetic field, using (4), we obtained

$$
u_{\alpha}=b_{a} E-D_{a} \frac{\nabla\left(n_{\alpha}\right)}{n_{\alpha}}
$$

where

$$
\begin{aligned}
b_{a} & =\frac{q_{\alpha}}{m_{\alpha} v_{\alpha n}} \\
D_{a} & =\frac{k T_{\alpha}}{m_{\alpha} v_{\alpha n}},
\end{aligned}
$$

where $D_{a}$ is transport coefficient. In this case (without magnetic field), as the electron mass is much smaller than the ion mass, the transport coefficient of electrons is much larger than that of the ions.

$$
D_{e} \gg D_{i} .
$$

In the case of magnetic field, using (8), the transport coefficient is expressed as

$$
D_{\alpha \perp}=\frac{v_{\alpha n} k T_{\alpha}}{m_{\alpha}\left(\omega_{\alpha}^{2}+v_{\alpha n}^{2}\right)} .
$$

In strong magnetic field $\left(\omega_{\alpha} \gg v_{\alpha n}\right)$, the diffusion coefficient can be written as follows:

$$
D_{\alpha \perp}=\frac{m_{\alpha} v_{\alpha n} k T_{\alpha}}{q_{\alpha}^{2} B^{2}} .
$$

From (57), the transverse transport coefficient of ions is larger than that of electrons.

$$
D_{i \perp}>D_{e \perp} .
$$

This conclusion is contrary to that without magnetic field. According to [18], in the absence of magnetic field, (35) is revised as

$$
a_{1}=\sqrt{\left(\frac{2.405}{R}\right)^{2} \frac{\left(D_{e \perp}-D_{i \perp}\right)}{\left(D_{e \|}-D_{i \|}\right)}-A^{2} p^{2}} .
$$

Substituting (58) into (59), the solution of (59) will be imaginary number, which is not allowed in the actual situation. Therefore, in the third part of this paper, we deduce the differential equations in magnetic field again according to the similar derivation process of the earlier articles.

Calculation analyses were carried out for discharge in magnetic field at pressures $p=10 \mathrm{~Pa}$, the distance between anode and cathode $l=0.02 \mathrm{~m}$, the parameter $A=5$ and $B_{c}=$ 19 , and anode plate voltage is $400 \mathrm{~V}$. The parameter of electron density $n_{a}$ is $10^{13} \mathrm{~m}^{-3}$.

Figure 2 is the axial distribution of the electron density under different magnetic fields. As can be seen from the diagram, the maximum value of the electron density distribution is at the anode. With the change of the cathode direction, the electron density decreases gradually. Within the range of $0 \mathrm{~m}$ to $0.01 \mathrm{~m}$, the electron density of the region is greatly affected by the magnetic field. Basically change exponentially the electronic density of the whole area, which is consistent with the theory of Townsend avalanche concept. Figure 3 is the axial distribution of ion density under different magnetic fields. In the process of the change from anode to cathode, the ion density distribution is consistent with the electron density distribution in the initial stage. Near the cathode, the ion density increases. Figure 4 shows the distribution of the electric field at different magnetic fields. The electric field is almost constant in the range of $0 \mathrm{~m}$ to $0.015 \mathrm{~m}$. The electric field increases gradually near the cathode. At the cathode layer, electrons are accelerated by the electric field. After a certain distance, the electrons reach enough energy and collide with neutral atoms. Under the action of electric field, the ions move towards the cathode region. So the cathode region is actually a region with a large positive ion concentration (Figure 2). According to the Poisson equation, a large electric field is formed by the slow motion of the positive ions (Figure 3).

In the process of discharge, electrons are subjected to the interaction of electric field and magnetic field. When the electric field is parallel to the magnetic field, the electron trajectory is not affected by the magnetic field. However, when the glow plasma is formed, due to the diffusion effect of the plasma, the confinement effect of the axial magnetic field 


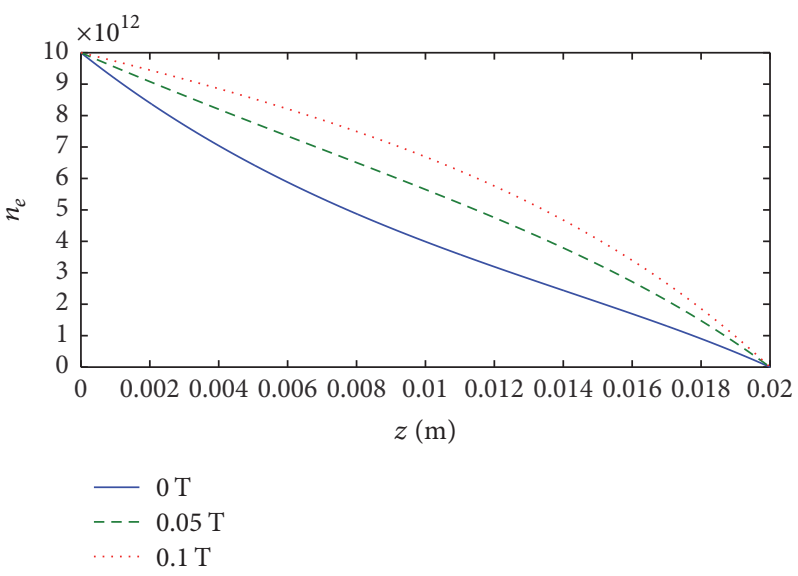

FIGURE 2: Axial electron density distribution under different magnetic fields.

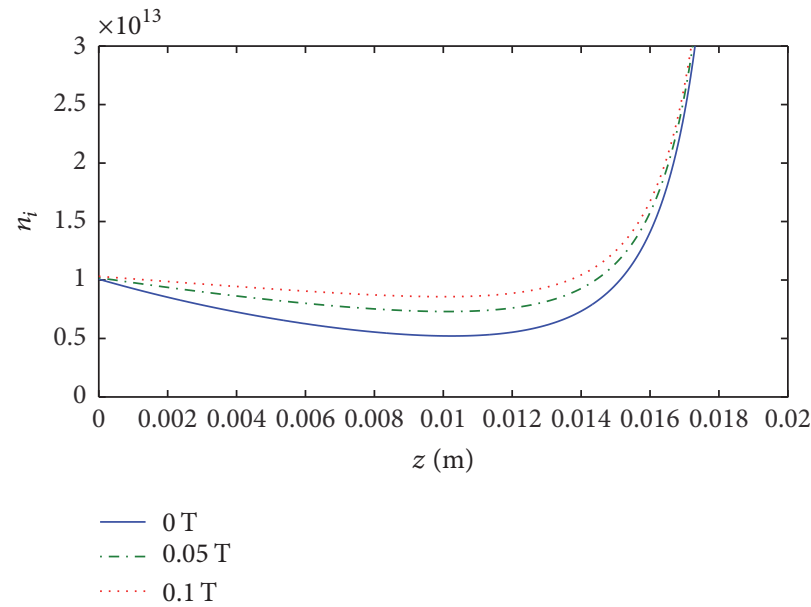

Figure 3: Axial ion density distribution under different magnetic fields.

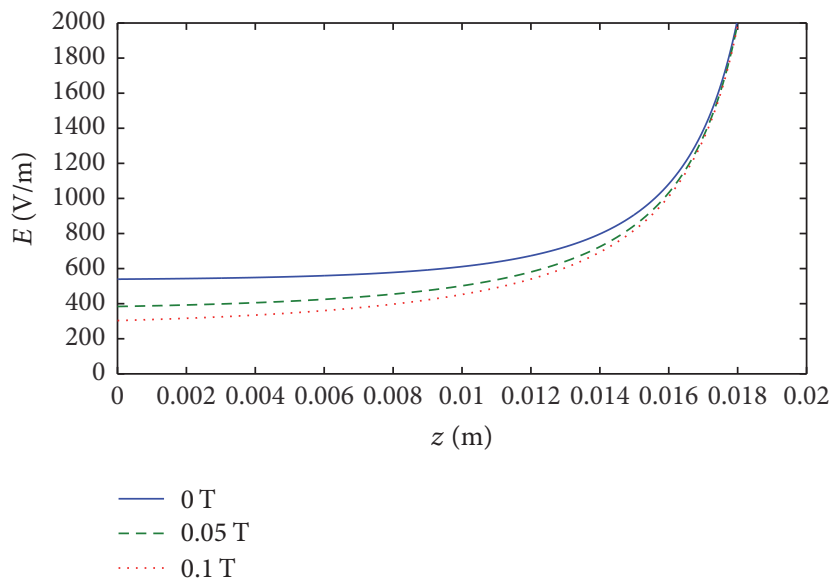

FIGURE 4: Electric field distribution under different magnetic fields.

on the plasma is reflected. The radial diffusion of plasma is suppressed. And this effect not only affects the radial component of the plasma, but also affects the axial distribution of the plasma parameters and the electric field distribution in the whole region. Figures 2 and 3 show that the electron and ion density increases with the magnetic field increases. In this model, the magnetic field does not increase the motion path of electrons. However, the confinement effect of the magnetic field on the charged particles in the plasma increases the collision probability between the free electrons 
and the neutral atoms in the unit area, thus increasing the plasma density.

\section{Conclusion}

The two-dimensional DC glow discharge model in axial magnetic field at low pressure was established. Based on a series of reasonable assumptions, the analytical solutions are obtained. It is shown that the model is consistent with the basic equations of the glow discharge without considering axial magnetic field, but the results are not the same. The results show that the magnetic field has a certain influence on the glow discharge parameters even when the magnetic field is parallel to the direction of the electric field.

\section{Conflicts of Interest}

The authors declare that there are no conflicts of interest regarding the publication of this paper.

\section{Acknowledgments}

This work is supported by the National Natural Science Foundation of China (no. 11075123), the Young Scientists Fund of Nature Science Foundation of China (no. 51207171), and Fundamental Research Funds for the Central Universities (no. 2042016kf1139).

\section{References}

[1] X. Zhao, S. Chen, K. Chen, and B. Chen, "Best magnetic condition to generate hollow cathode glow plasma in high vacuum," Plasma Science and Technology, vol. 16, no. 1, pp. 21-25, 2014.

[2] C. D. Cothran, D. R. Boris, C. S. Compton et al., "Continuous and pulsed electron beam production from an uninterrupted plasma cathode," Surface \& Coatings Technology, vol. 267, pp. 111-116, 2014.

[3] A. S. Metel, S. N. Grigoriev, M. A. Volosova, V. P. Bolbukov, and Y. A. Melnik, "Role of electrostatic and magnetic electron confinement in a hollow-cathode glow discharge in a nonuniform magnetic field," Plasma Physics Reports, vol. 41, no. 2, pp. 188197, 2015.

[4] M. Hu, S. D. Wan, L. Zhong, H. Liu, and H. Wang, "Magnetic control of the constant-current glow discharge plasma characteristics," Acta Physica Sinica, vol. 61, no. 4, Article ID 045201, 2012.

[5] I. M. Ulanov and A. Y. Litvintsev, "Experimental study of the effect of a longitudinal magnetic field on the cathode parts of a glow discharge in helium," Technical Physics, vol. 49, no. 9, pp. 1135-1141, 2004.

[6] J. E. Harry and D. R. Evans, "Magnetic stabilization of a rotating glow discharge," Journal of Applied Physics, vol. 62, no. 12, pp. 4708-4711, 1987.

[7] Y. Fu, H. Luo, X. Zou, and X. Wang, "Validity of the similarity law for the glow discharges in non-plane-parallel gaps," Plasma Sources Science and Technology, vol. 23, no. 6, Article ID 065035, 6 pages, 2014.

[8] M. N. Stankov and M. D. Petkovic, "Numerical modelling of dc argon glow discharge at low pressure without and with $\operatorname{Ar}\left({ }^{3} \mathrm{P}_{2}\right)$ metastable state," Romanian Journal of Physics, vol. 59, no. 3-4, pp. 328-338, 2014.

[9] S. T. Surzhikov and J. S. Shang, "Numerical simulation of subsonic gas flows with glow discharge and magnetic field," in Proceedings of the 34th AIAA Plasmadynamics and Lasers Conference, Oriando, Fla, USA, June 2003.

[10] A. V. Isakov, V. P. Kolesnik, A. M. Okhrimovskyy, N. P. Stepanushkin, and A. A. Taran, "Computer simulation of abnormal glow discharge processes in crossed electric and magnetic fields," Problems of Atomic Science and Technology, vol. 6, no. 20, pp. 171-174, 2014.

[11] A. S. Petrusev and S. T. Surzhikov, "Efficient algorithm for simulating a multidimensional glow discharge," Plasma Physics Reports, vol. 34, no. 3, pp. 239-244, 2008.

[12] Y. Wang, Y. Liu, S. Zheng, and G. Lin, "Effect of RF on RF nitrogen discharge with induced argon plasma at high pressure," Journal of Plasma Physics, vol. 78, no. 6, pp. 673-676, 2012.

[13] S. Surzhikov and J. Shang, "Normal glow discharge in axial magnetic field," Plasma Sources Science and Technology, vol. 23, no. 5, Article ID 054017, 8 pages, 2014.

[14] S. T. Surzhikov and J. S. Shang, "Two-component plasma model for two-dimensional glow discharge in magnetic field," Journal of Computational Physics, vol. 199, no. 2, pp. 437-464, 2004.

[15] S. T. Pai, "Analytic approach to glow discharge theory: the physical model," Journal of Applied Physics, vol. 71, no. 12, pp. 5820$5825,1992$.

[16] S. T. Pai and X. M. Guo, "Analytic approach to glow discharge theory: result and analysis," Journal of Applied Physics, vol. 71, no. 12 , pp. 5826-5833, 1992.

[17] G. Xiao-Ming and S. T. Pai, "A mathematical procedure for modelling low temperature plasma," Acta Physica Sinca, vol. 44, no. 4, pp. 565-569, 1995 (Chinese).

[18] S. T. Pai, X. M. Guo, and T. D. Zhou, "Closed form analytic solutions describing glow discharge plasma," Physics of Plasmas, vol. 3, no. 10, pp. 3842-3852, 1996.

[19] S. T. Pai and Z. Ting-Dong, "Theoretical analysis of current distributions of glow discharge plasma in 3-dimensional space," Acta Physica Sinica, vol. 42, no. 9, pp. 1463-1470, 1993 (Chinese). 


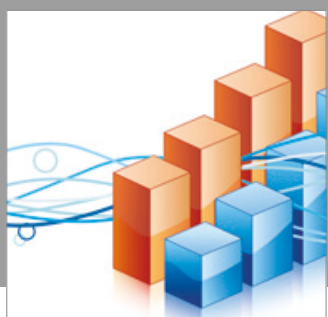

Advances in

Operations Research

vatersals

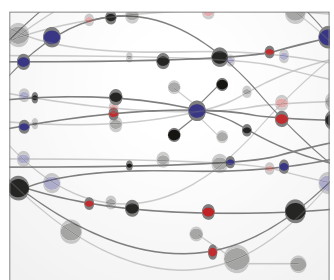

\section{The Scientific} World Journal
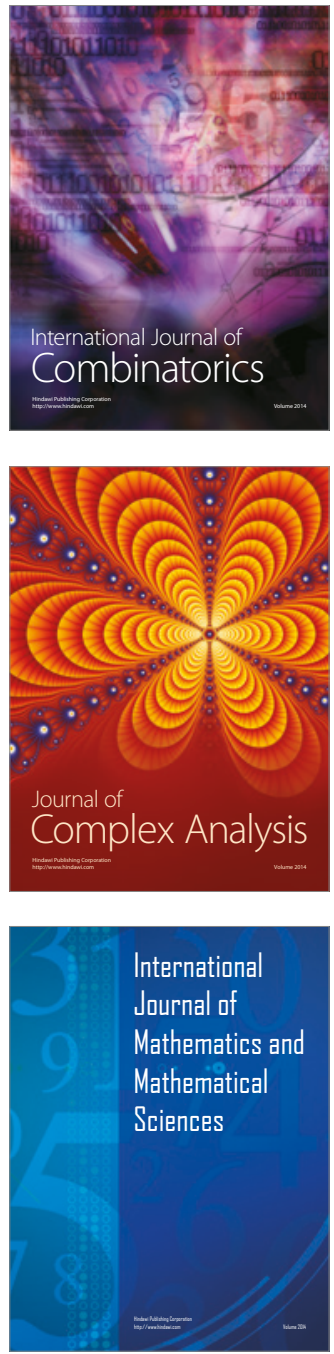
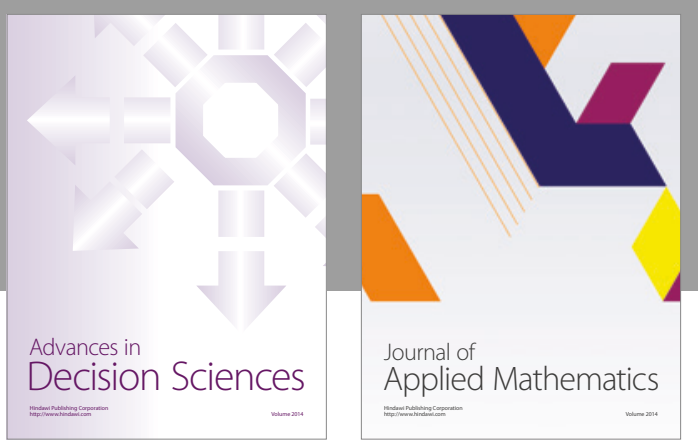

Algebra

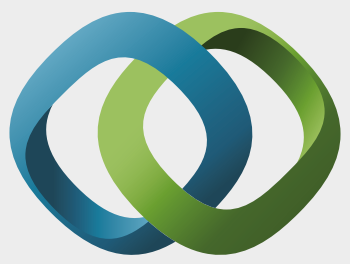

\section{Hindawi}

Submit your manuscripts at

https://www.hindawi.com
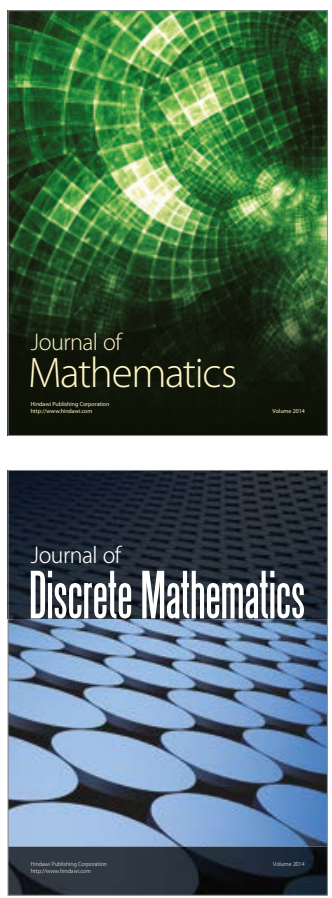

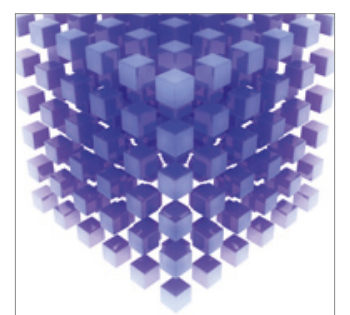

Mathematical Problems in Engineering
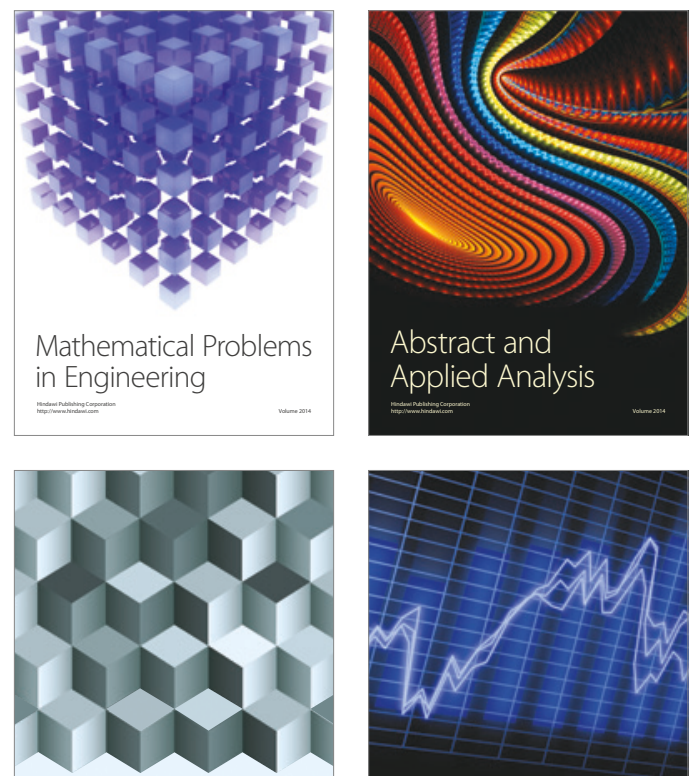

Journal of

Function Spaces

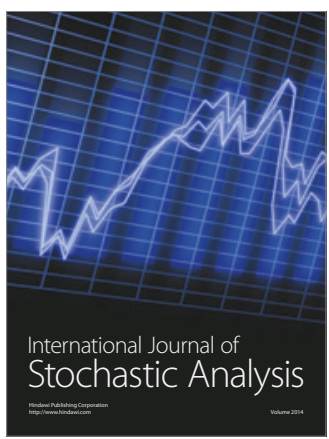

Probability and Statistics
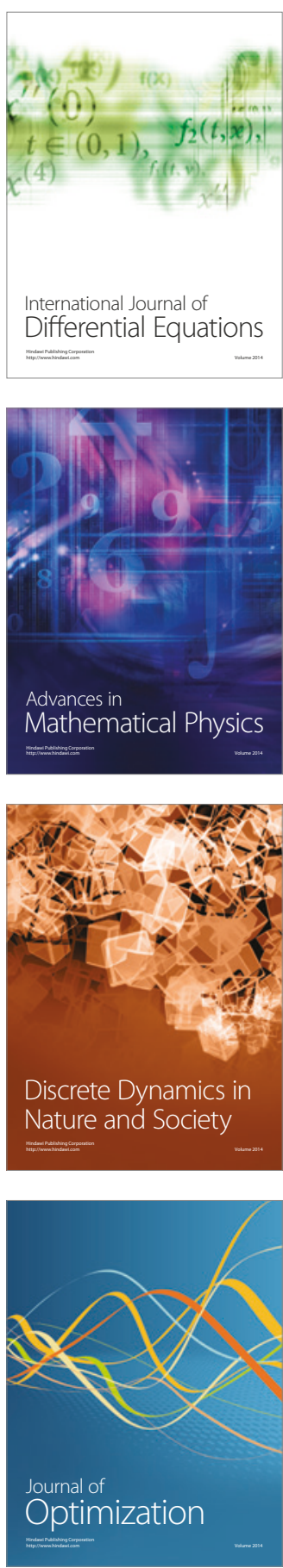